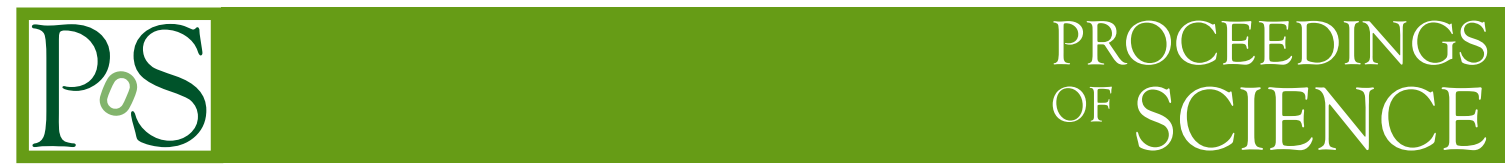

\title{
Electroweak corrections to top pair production near threshold
}

\section{Andreas Maier*}

Institute for Particle Physics Phenomenology, Department of Physics, Durham University, Durham DH1 3LE, United Kingdom

E-mail: andreas.maieredurham.ac.uk

I review the impact of electroweak corrections on the total production cross section of a nonrelativistic top-antitop pair at a future electron-positron collider. I outline the implications for the measurement of the top quark mass, width, and Yukawa coupling.

Loops and Legs in Quantum Field Theory

24-29 April 2016

Leipzig, Germany

\footnotetext{
${ }^{*}$ Speaker.
} 


\section{Introduction}

The properties of the top quark can be measured with unprecedented precision at a future electron-positron collider, like the proposed International Linear Collider [1]. A key quantity in this respect is the total production cross section near the top-antitop threshold.

In order to match the projected experimental precision, it is mandatory to take into account the combined effects of bound-state interactions and higher-order perturbative corrections. These effects are incorporated systematically in the effective field theory framework of potential nonrelativistic quantum chromodynamics (PNRQCD) [2]. Within PNRQCD, the cross section is expanded simultaneously in the small non-relativistic velocity $v$ and the strong coupling constant $\alpha_{s}$, adopting the power counting $\alpha_{s} \sim v \ll 1$. The exchange of Coulomb gluons yields contributions scaling with powers of $\alpha_{s} / v \sim 1$, which are resummed to all orders.

The PNRQCD Lagrangian at a given order can be derived systematically by matching to nonrelativistic quantum chromodynamics (NRQCD) [3], which in turn is obtained by matching to the fundamental theory of QCD. ${ }^{1}$ The top-antitop production cross section within PNRQCD has been computed to third order [4]; the power counting up to this order is given by

$$
\sigma \sim v \sum_{k}\left(\frac{\alpha_{s}}{v}\right)^{k} \times\left\{\begin{array}{ll}
1 & \mathrm{LO} \\
\alpha_{s}, v & \mathrm{NLO} \\
\alpha_{s}^{2}, \alpha_{s} v, v^{2} & \mathrm{~N}^{2} \mathrm{LO} \\
\alpha_{s}^{3}, \alpha_{s}^{2} v, \alpha_{s} v^{2}, v^{3} & \mathrm{~N}^{3} \mathrm{LO}
\end{array} .\right.
$$

Corrections beyond QCD can be taken into account by generalising the aforementioned twostep matching procedure to the case where the fundamental theory is given by the full Standard Model. It is customary to adopt the power counting $\alpha \sim \alpha_{s}^{2}$ for the QED coupling constant $\alpha$. For the Higgs sector, we will count powers of the Yukawa coupling to be of the same order as the strong coupling constant, i.e. $y_{t} \sim \alpha_{s}$. The value of the Higgs boson mass $m_{H}$ lies between the hard scale given by the top quark mass $m_{t}$ and its nonrelativistic momentum $m_{t} v$, which defines the soft scale. Since the numerical value is much closer to the former, we use the power counting $m_{H} \sim m_{t}$.

\section{Classification of electroweak corrections}

In the following we will consider different classes of effects beyond QCD. Apart from the electroweak corrections to the production all of them are discussed in more detail in [5].

\subsection{Higgs effects}

In a first step, we incorporate Higgs effects by adding

$$
\mathscr{L}_{\text {Higgs }}=\frac{1}{2}\left(\partial_{\mu} H\right)^{2}-\frac{1}{2} m_{H}^{2} H^{2}-\sqrt{\frac{\lambda}{2}} m_{H} H^{3}-\frac{\lambda}{4} H^{4}-\frac{y_{t}}{\sqrt{2}} \bar{t} t H
$$

with $\lambda=\frac{\pi \alpha m_{H}^{2}}{2 m_{W}^{2} s_{W}^{2}}$ to the QCD Lagrangian. Corrections then arise from the exchange of a virtual Higgs boson between the top and the antitop (see figure 1).

\footnotetext{
${ }^{1}$ At electron-positron colliders, quark-antiquark pairs are mainly produced via a virtual photon or $\mathrm{Z}$ boson. Thus, the corresponding vertices should also be added to the QCD Lagrangian.
} 

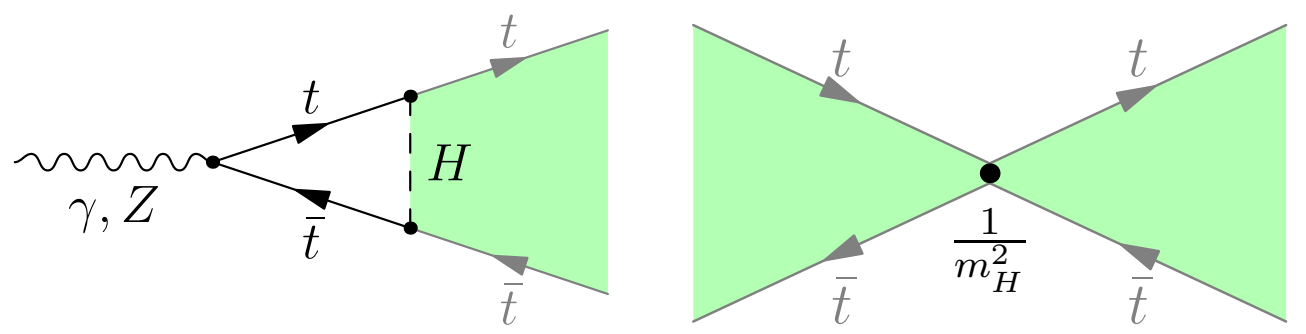

Figure 1: Example diagrams for Higgs corrections to $t \bar{t}$ production. Left: $\mathrm{N}^{2} \mathrm{LO}$ correction to the production vertex. Right: $\mathrm{N}^{3} \mathrm{LO}$ potential correction. Grey lines indicate nonrelativistic (anti-)top quarks near their mass shell. The shaded area represents the leading-order colour Coulomb interaction.

A Higgs boson with a hard momentum (of the order of the Higgs boson mass), can only be exchanged if the initially produced top-antitop pair is far off shell. Within the effective theory, this constitutes a correction to the production vertex of the nonrelativistic top-antitop pair. A single Higgs exchange contributes with a factor of $y_{t}^{2}$ and is thus a $\mathrm{N}^{2} \mathrm{LO}$ correction. We also take into account the exchange of an additional gluon, which contributes at $\mathrm{N}^{3} \mathrm{LO}$. These corrections were calculated in $[6,7,8,9]$.

For a Higgs exchange between a top-antitop pair near mass shell, the Higgs momentum $\mathbf{q} \sim$ $m_{t} v$ is much smaller than its mass and thus can be neglected inside the Higgs propagator. The Higgs exchange then corresponds to a local correction $\delta_{H} V=-y_{t}^{2} /\left(2 m_{t}^{2}\right) \sim v^{2} / m_{t}^{2}$ to the interaction potential. The leading-order colour Coulomb potential is proportional to $\alpha_{s} / \mathbf{q}^{2} \sim 1 /\left(v m_{t}^{2}\right)$. Thus, the Higgs potential is suppressed by a factor $v^{3}$ with respect to the leading-order potential and first contributes at $\mathrm{N}^{3} \mathrm{LO}$.

\subsection{QED Coulomb potential}

In order to take into account QED corrections, we add

$$
\mathscr{L}_{\mathrm{QED}}=-\frac{1}{4} F_{\mu v} F^{\mu v}+\sum_{l \in \text { leptons }} \bar{\psi}_{l} i \not \partial \psi_{l}-\sum_{f \in \text { fermions }} e_{f} \bar{\psi}_{f} A \psi_{f}
$$

to the Lagrangian. The dominant correction is then given by the QED Coulomb potential $\alpha / \mathbf{q}^{2}$, which is a NLO effect.

\subsection{Nonresonant production}

Since the top quark is unstable, its decay into a $W^{+} b$ pair has to be incorporated consistently into our framework. Within unstable particle effective theory [10,11], the cross section is given as a sum of resonant top-antitop production and nonresonant production including top decays. At NLO it suffices to consider nonresonant production of the final states $t W^{-} \bar{b}$ and $\bar{t} W^{+} b$, which was computed in [12]. The diagrams contributing to the latter final state are shown in figure 2.

\subsection{P-wave production}

Due to the axialvector coupling of the $\mathrm{Z}$ boson the top-antitop pair can be produced in a $\mathrm{P}$ wave state. P-wave production starts to contribute at $\mathrm{N}^{2} \mathrm{LO}$ and has been found to be numerically small $[13,14,15,16]$. 

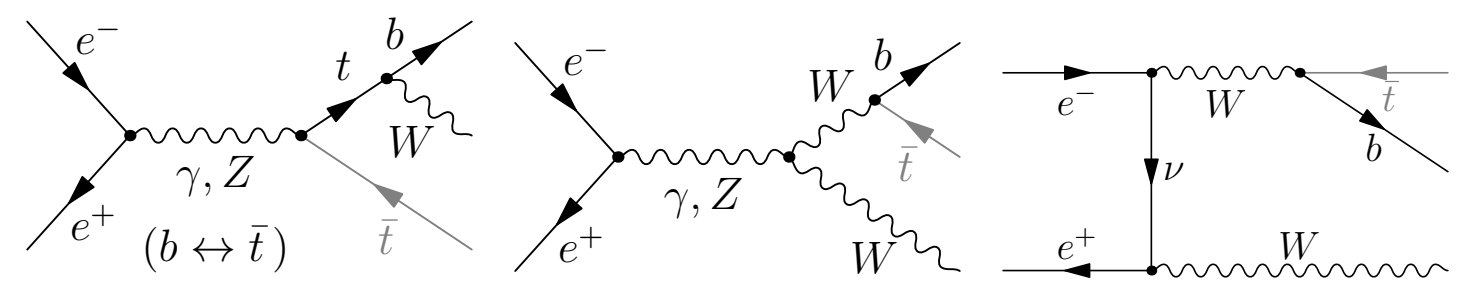

Figure 2: Non-resonant production of $\bar{t} W^{+} b$. Grey lines indicate on-shell antitop quarks.

\subsection{Electroweak production corrections}

At $\mathrm{N}^{2} \mathrm{LO}$, the full Standard Model Lagrangian induces further corrections to the resonant topantitop production, which have been calculated in $[6,7,17,8]$. Sample diagrams are shown in figure 3. To obtain consistent predictions, these have to be combined with the $\mathrm{N}^{2} \mathrm{LO}$ nonresonant production, where only partial results are known [18, 19, 20].
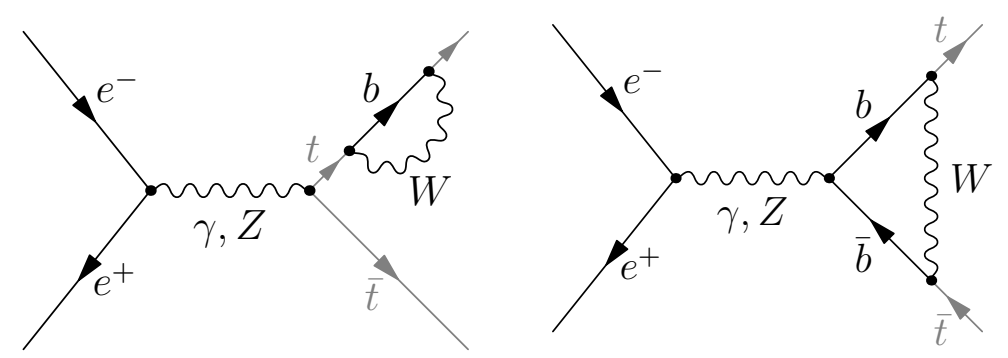

Figure 3: Sample diagrams contributing to the electroweak production corrections. Grey lines indicate on-shell (anti-)top quarks.

\section{Phenomenological impact}

Figure 4 demonstrates the combined effect of the corrections discussed in section 2 on the total top-antitop production cross section. Excluding the electroweak production corrections outlined in section 2.5, we see a notable change in the cross section of up to $10 \%$. Especially in the peak region the change is significantly larger than the pure QCD scale uncertainty. Once we include the $\mathrm{N}^{2} \mathrm{LO}$ electroweak corrections (excluding $\mathrm{N}^{2} \mathrm{LO}$ nonresonant production), the cross section drops again visibly, to the level of the pure QCD result or even below. While the current "full" result does not correspond to a fully consistent theory description, it may indicate the magnitude of the combined $\mathrm{N}^{2} \mathrm{LO}$ nonresonant and electroweak production corrections.

In figure 5 we compare the theory uncertainty estimated from scale variation between $50 \mathrm{GeV}$ and $350 \mathrm{GeV}$ to the change in the cross section upon variation of various input parameters. The size and shape of the variation suggests that the top-quark mass in the potential-subtracted (PS) scheme [21] can be determined to an accuracy of better than $100 \mathrm{MeV}$ at a future high-energy electron-positron collider. This estimate is backed up by a recent preliminary experimental analysis [22]. In contrast, the cross section is not very sensitive to modifications of the Yukawa coupling and the change in the shape is hard to distinguish from the one induced by a variation of the strong coupling constant. 

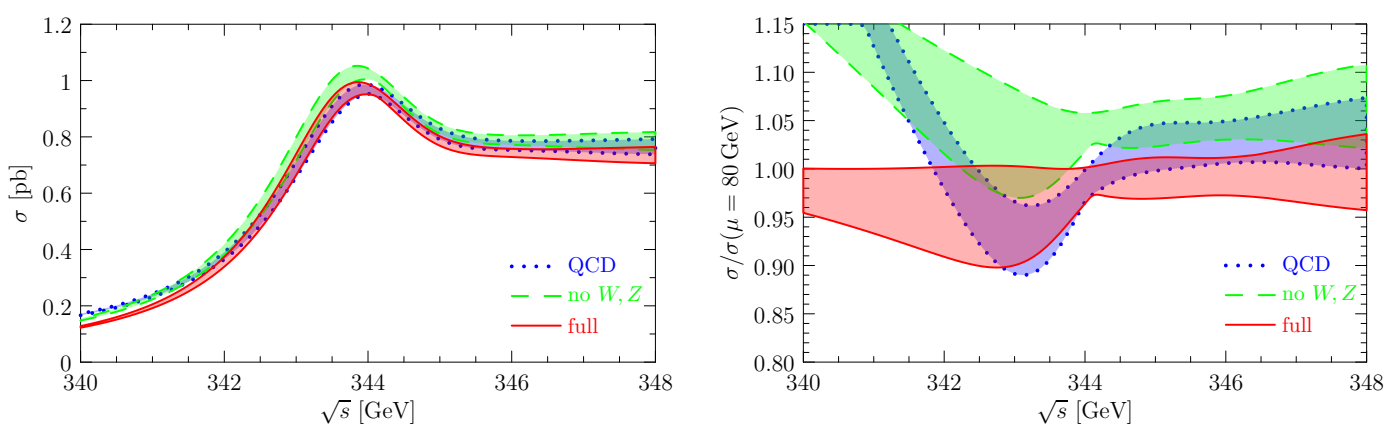

Figure 4: Cross section for $t \bar{t}$ production with $m_{t}^{\mathrm{PS}}(20 \mathrm{GeV})=171.5 \mathrm{GeV}, \Gamma_{t}=1.33 \mathrm{GeV}, m_{H}=$ $125 \mathrm{GeV}, \alpha_{s}\left(m_{Z}\right)=0.1185, \alpha\left(m_{Z}\right)=1 / 128.944$. Error bands result from scale variation between $50 \mathrm{GeV}$ and $350 \mathrm{Gev}$. The dotted blue line shows the $\mathrm{N}^{3} \mathrm{LO}$ QCD-only cross section, whereas for the dashed green line also Higgs, QED, and non-resonant corrections are included. The solid red line includes all known corrections. On the right, the cross section is normalized to the full cross section at a scale of $80 \mathrm{GeV}$.
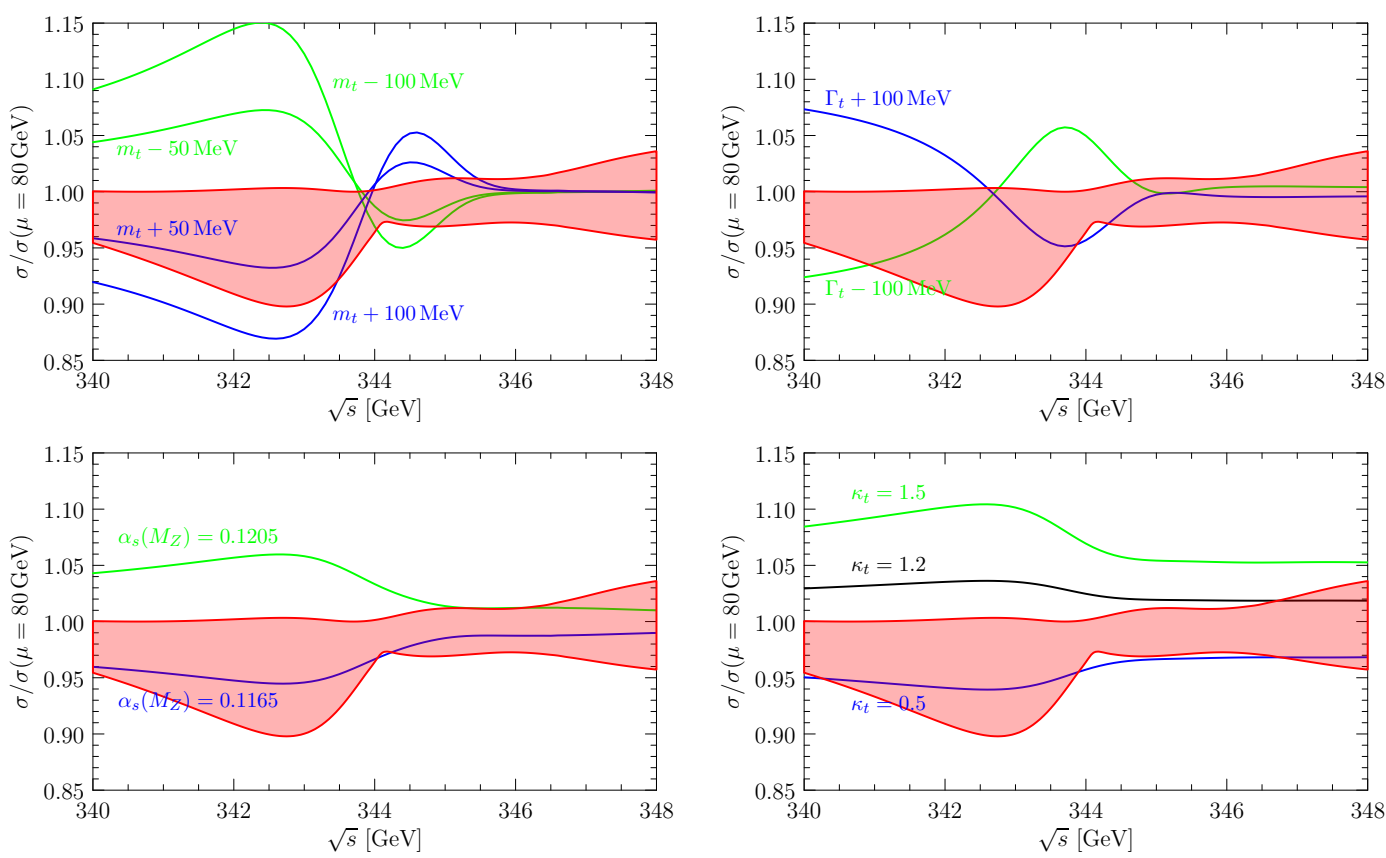

Figure 5: Change of the $t \bar{t}$ cross section upon variation of the top quark mass (upper left), width (upper right), strong coupling (lower left), and top Yukawa coupling $y_{t}$ with $\kappa_{t}=y_{t} / y_{t}^{\mathrm{SM}}$ (lower right). The shaded band corresponds to the normalised full cross section shown in figure 4.

\section{Conclusion}

For the determination of the properties of the top quark at a future electron-positron collider a precise theory prediction is vital. Effects beyond QCD can be as big as $10 \%$ and exceed the unertainty of the $\mathrm{N}^{3} \mathrm{LO}$ QCD prediction. The top-quark mass can be extracted from the cross section with an uncertainty of presumably less than $100 \mathrm{MeV}$ and an equally accurate measurement of the top-quark width seems also feasible. The sensitivity to the top Yukawa coupling is small and its determination will require a very precise knowledge of the value of the strong coupling constant. 
The code employed for the presented analysis has been made public [23] and can be downloaded from https://www.hepforge.org/downloads/qqbarthreshold/.

\section{Acknowledgements}

A.M. is supported by a European Union COFUND/Durham Junior Research Fellowship under EU grant agreement number 267209. This work was supported by the DFG Sonderforschungsbereich/Transregio 9 "Computergestützte Theoretische Teilchenphysik", the Gottfried Wilhelm Leibniz programme of the Deutsche Forschungsgemeinschaft (DFG) and the DFG cluster of excellence "Origin and Structure of the Universe".

\section{References}

[1] H. Baer, T. Barklow, K. Fujii, Y. Gao, A. Hoang, S. Kanemura et al., The International Linear Collider Technical Design Report - Volume 2: Physics, 1306.6352.

[2] A. Pineda and J. Soto, Effective field theory for ultrasoft momenta in NRQCD and NRQED, Nucl. Phys. Proc. Suppl. 64 (1998) 428-432, [hep-ph/9707481].

[3] W. E. Caswell and G. P. Lepage, Effective Lagrangians for Bound State Problems in QED, QCD, and Other Field Theories, Phys. Lett. B167 (1986) 437-442.

[4] M. Beneke, Y. Kiyo, P. Marquard, A. Penin, J. Piclum and M. Steinhauser, Next-to-Next-to-Next-to-Leading Order QCD Prediction for the Top Antitop S-Wave Pair Production Cross Section Near Threshold in $e^{+} e^{-}$Annihilation, Phys. Rev. Lett. 115 (2015) 192001, [1506.06864].

[5] M. Beneke, A. Maier, J. Piclum and T. Rauh, Higgs effects in top anti-top production near threshold in $e^{+} e^{-}$annihilation, Nucl. Phys. B899 (2015) 180-193, [1506.06865].

[6] B. Grządkowski, J. H. Kühn, P. Krawczyk and R. G. Stuart, Electroweak Corrections on the Toponium Resonance, Nucl. Phys. B281 (1987) 18.

[7] R. J. Guth and J. H. Kühn, Top quark threshold and radiative corrections, Nucl. Phys. $\mathbf{3 3 6 8}$ (1992) $38-56$.

[8] A. H. Hoang and C. J. Reißer, On electroweak matching conditions for top pair production at threshold, Phys. Rev. D74 (2006) 034002, [hep-ph/ 0604104 ].

[9] D. Eiras and M. Steinhauser, Complete Higgs mass dependence of top quark pair threshold production to order $\alpha \alpha_{s}$, Nucl. Phys. $\mathbf{B} 757$ (2006) 197-210, [hep-ph/ 0605227 ].

[10] M. Beneke, A. P. Chapovsky, A. Signer and G. Zanderighi, Effective theory approach to unstable particle production, Phys. Rev. Lett. 93 (2004) 011602, [hep-ph/0312331].

[11] M. Beneke, A. P. Chapovsky, A. Signer and G. Zanderighi, Effective theory calculation of resonant high-energy scattering, Nucl. Phys. B686 (2004) 205-247, [hep-ph/ 0401002 ].

[12] M. Beneke, B. Jantzen and P. Ruiz-Femenía, Electroweak non-resonant NLO corrections to $e^{+} e^{-} \rightarrow W^{+} W^{-} b \bar{b}$ in the $t \bar{t}$ resonance region, Nucl. Phys. B840 (2010) 186-213, [1004.2188].

[13] I. I. Y. Bigi, V. S. Fadin and V. A. Khoze, Stop near threshold, Nucl. Phys. B377 (1992) 461-479.

[14] A. A. Penin and A. A. Pivovarov, Top quark threshold production in gamma gamma collision in the next-to-leading order, Nucl. Phys. B550 (1999) 375-396, [hep-ph/ 9810496 ]. 
[15] A. A. Penin and A. A. Pivovarov, Analytical results for $e^{+} e^{-} \rightarrow t \bar{t}$ and $\gamma \gamma \rightarrow t \bar{t}$ observables near the threshold up to the next-to-next-to leading order of NRQCD, Phys. Atom. Nucl. 64 (2001) 275-293, [hep-ph/9904278].

[16] M. Beneke, J. Piclum and T. Rauh, P-wave contribution to third-order top-quark pair production near threshold, Nucl. Phys. B880 (2014) 414-434, [1312 . 4792].

[17] A. H. Hoang and C. J. Reißer, Electroweak absorptive parts in NRQCD matching conditions, Phys. Rev. D71 (2005) 074022, [hep-ph/ 0 412258].

[18] A. A. Penin and J. H. Piclum, Threshold production of unstable top, JHEP 01 (2012) 034, [1110.1970].

[19] B. Jantzen and P. Ruiz-Femenía, Next-to-next-to-leading order nonresonant corrections to threshold top-pair production from $e^{+} e^{-}$collisions: Endpoint-singular terms, Phys.Rev. D88 (2013) 054011, [1307.4337].

[20] P. Ruiz-Femenía, First estimate of the NNLO nonresonant corrections to top-antitop threshold production at lepton colliders, Phys. Rev. D89 (2014) 097501, [1402.1123].

[21] M. Beneke, A quark mass definition adequate for threshold problems, Phys. Lett. $\mathbf{B 4 3 4}$ (1998) 115-125, [hep-ph/9804241].

[22] F. Simon, A First Look at the Impact of NNNLO Theory Uncertainties on Top Mass Measurements at the ILC, in International Workshop on Future Linear Colliders (LCWS15) Whistler, B.C., Canada, November 2-6, 2015, 2016. 1603.04764.

[23] M. Beneke, Y. Kiyo, A. Maier and J. Piclum, Near-threshold production of heavy quarks with QQbar_threshold, 1605.03010. 